\title{
Combined Sales Effort and Inventory Control under Demand Uncertainty
}

\author{
Weili Xue, ${ }^{1}$ Xiaolin $\mathrm{Xu}^{2}{ }^{2}$ and Ruxian Wang ${ }^{3}$ \\ ${ }^{1}$ School of Management and Engineering, Nanjing University, Nanjing 210093, China \\ ${ }^{2}$ School of Business, Nanjing University, Nanjing 210093, China \\ ${ }^{3}$ Hewlett-Packard Laboratories, Hewlett-Packard Co., Palo Alto, CA 94304, USA
}

Correspondence should be addressed to Xiaolin Xu; xuxl@nju.edu.cn

Received 9 December 2012; Accepted 19 December 2012

Academic Editor: Xiang Li

Copyright (C) 2013 Weili Xue et al. This is an open access article distributed under the Creative Commons Attribution License, which permits unrestricted use, distribution, and reproduction in any medium, provided the original work is properly cited.

\begin{abstract}
We study the joint inventory and sales effort management problems of a retailer in a broad context and investigate the optimal policies for a single item, periodic-review system. In each period, the demand is uncertain depending on the sales effort level exerted by the retailer, which incurs an associated cost. The retailer's objective is to find a joint optimal inventory replenishment and sales effort policy to maximize the discounted profit over a finite horizon. We first consider a basic setting with zero setup cost and no batch ordering, under which the base stock list sales effort policy is optimal. Two extensions are then investigated: (1) the case with nonzero setup cost, under which we show that $(s, S, e)$ policy is optimal; and (2) the case with batch ordering, under which we prove the optimality of the $(r, N q, e)$ policy. Finally, we conduct numerical studies to provide additional managerial insights.
\end{abstract}

\section{Introduction}

Inventory and demand management has received much attention due to the increasingly competitive environment. Traditional inventory problems often consider an exogenously determined uncertain demand and thus the key decision facing firms is to decide on an appropriate inventory policy to mitigate the mismatch cost of demand and supply. However, demand could be endogenously influenced by many factors such as price, freshness level of the product, and the sales effort exerted by firms. Within these factors, changing sales effort level is an important driver to match supply and demand and achieve business goals. In this paper, we focus on the optimal decision on the sales effort which includes, to list a few, the incentives to the sales people and the promotions with short-term effects such as in-store displays, product-service bundling, and so forth.

Specifically, we study the joint inventory and sales effort management of a retailer in a broad context and investigate his/her optimal policies under a single item, periodicreview system. In each period, the retailer decides on the replenishment quantity and the sales effort to be exerted jointly. For the basic setting without considering the setup cost and batch ordering, we assume that the procurement cost is linearly increasing in the order quantity and the associated cost for exerting sales effort (e.g., rentals for instore displays and advertisement expenditure in media like TV and newspaper) is increasing and convex in the sales effort level. All replenishment arrives immediately and all unmet demand is fully backlogged. The inventory holding and shortage cost are charged based on the inventory leftover at the end of each period. The retailer's objective is to find a joint optimal inventory replenishment and sales effort policy to maximize the discounted profit over a finite horizon. In addition to the basic setting mentioned above, we will go further to investigate the retailer's optimal polices when each ordering incurs a non-zero setup cost and when the order quantity for each procurement must be in batch size respectively.

The model studied in this paper is closely related to those dealing with the coordination between marketing and operations management in general and those studying the jointly pricing and inventory control problem under a multi period framework in particular. Here we briefly review 
the most relevant work. As to the literature on joint pricing and inventory control, reader of interest can refer to Yano and Gilbert [1] for detailed reviews. Karakul [2], Serel [3], and Webster and Weng [4] study these problems with a single period setting. In a multi period setting, when there is no ordering setup cost, Federgruen and Heching [5] show the optimality of the so-called base stock list price policy. When there is a setup cost for each ordering, several authors have identified the conditions under which an $(s, S, p)$ policy is optimal. In a finite horizon periodic-review setting, Chen and Simchi-Levi [6] show the optimality of the $(s, S, p)$ policy or a variation of such a policy. Chen and Simchi-Levi [7] extend the optimality of a stationary $(s, S, p)$ policy to an infinite horizon setting. The sales effort considered in our paper is different from the pricing decisions, as it will not influence the selling price of the product. Such sales effort is rather reasonable as the retailers tend to avoid frequent price changes, which on one hand may bring down the customer's perception on the product, and on the other hand often incur additional cost (see, e.g., Chen et al. [8]).

Our research is also closely related to literature investigating the impact of various sales efforts on operational inventory decisions. Balcer [9] consider a joint inventory and advertising strategy problem. Cheng and Sethi [10] study the joint inventory-promotion problem with Markov-dependent demand state. Porteus and Whang [11] and Chen [12] analyze the impact of incentive schemes of sales force compensation on manufacturing decisions. Ernst and Kouvelis [13] study the joint decision of goods bundling and inventory control in a newsvendor setting. In a continuous-review setting, Chen et al. [14] show that the $(s, S)$ type policy is optimal for product inventory control, and an inventory level-based service package composite is optimal for service offerings. Zhang et al. [15] discuss the joint optimization of inventory and pricing, and promotion, and ignore the fixed ordering cost in their setting. Wei and Chen [16] study the computation method for the joint inventory and sales effort model in an infinite horizon framework, when the optimal policy has an $(s, S, e)$ type. However, to the best of our knowledge, few researchers have been devoted to study the optimal joint inventory and sales effort policy under a periodic-review setting.

Our current work shares some similarities with Wei and Chen [16]. However, the model is different from theirs in the following ways. First, our model bases on the finite horizon setting and the system parameters can be nonstationary, while their analysis focuses on the infinite horizon with stationary policy. Second, we derive the optimal policy, while they only optimize the policy parameters given the policy form. Third, we study the case when the demand has a multiplicative form with the sales effort level. Moreover, we also extend the analysis to the case when the order quantity is in batch size. Through our analysis, we find that the optimal policy is a base stock list sales effort policy when the setup cost is negligible and is a $(s, S, e)$ policy when the setup cost is considered. When the order size in each period is in batch, we show the optimality of a $(r, N q, e)$ policy when there is no setup cost. Numerical study shows that both the base stock level and the optimal sales effort level are decreasing with the unit cost and the diseconomy of scale of the sales effort cost. However, although the base stock level is increasing in the demand uncertainty, the corresponding sales effort level is decreasing, which implies that the cost caused by additional demand variance is larger than the profits made by additional expected demand when the demand has a multiplicative form in the sales effort level.

The reminder of this paper is organized as follows. In Section 2, we first setup and analyze the basic model in which there is no setup cost for each ordering. Then, in Section 3, we extend the analysis to the case when there is a setup cost and the case when the order size is in batch, respectively. The optimal policies for both cases are identified. In Section 4, we employ a numerical study to gain more insights on the joint inventory and sales effort problem. Section 5 concludes the paper with possible future directions.

\section{Model Formulation}

Consider a multi period inventory planning problem faced by a retailer with sales effort sensitive demand. There are $T$ periods. At the beginning of each period $t, t=1,2, \ldots, T$, depending on his/her on hand inventory level $x_{t}$, the retailer should decide how many to order from the supplier at a linear unit cost $c_{t}$ (actually, we will extend our analysis to the case when there is a setup cost for each procurement) and how much sales effort, $e_{t}$, to exert to stimulate the demand. The replenishment becomes available instantaneously; that is, the lead time is zero. Let $y_{t}$ be the inventory level after orders arrive. The cost to exert effort $e_{t}$ is $v\left(e_{t}\right)$, which is increasing convex in $e_{t}$ with $v(1)=0$. That is, the marginal cost to exert the effort is increasing in the effort level. The new demand after exerting sales effort $e_{t}$ is given by $e_{t} \xi$, in which $\xi$ is a random variable when there is no sales effort exerts (i.e., $e=$ 1). Thus, the sales effort, although will increase the expected sales, will also bring up the variance of the demand; however, the coefficient of variant of the demand keeps constant. Let $F(\cdot)$ and $f(\cdot)$ be the cumulative distribution function and the probability density function of $\xi$, respectively. Without loss of generality, the demands in consecutive periods are assumed to be independent and nonnegative.

The unit selling price of the product, $p$, is exogenously given. Such exogenously determined selling price reflects the retailer's limited pricing power when facing intense competition in the market. The demand realizes. If demand exceeds the available inventory, unsatisfied demand is backlogged and incurs shortage cost; otherwise, excess inventory incurs holding cost and will be carried to the next period. Let $H_{t}(I)$ be the holding or backlogging cost incurred in period $t$ with ending inventory level $I$. Let $L_{t}(y, e)=E\left[H_{t}\left(y-e \xi_{t}\right)\right]$ denote the one-period expected inventory and backlogging costs for period $t$, when the inventory level after order arrives is $y$ and the exerted sales effort is $e$. We impose the following assumption on the function $L_{t}(y, e)$ and $H_{t}(I)$.

Assumption 1. (i) $\lim _{y \rightarrow \infty} L_{t}(y, e)=\lim _{y \rightarrow-\infty}\left[c_{t} y+\right.$ $\left.L_{t}(y, e)\right]=\lim _{y \rightarrow \infty}\left[\left(c_{t}-\alpha c_{t+1}\right) y+L_{t}(y, e)\right]=\infty$ for all $e \in\left[e_{\min }, e_{\max }\right]$; (ii) $H_{t}(I)$ is convex in $I$. 
The first part of this assumption holds whenever the inventory (and backlogging) cost function, $L_{t}$, tends to infinity as the inventory level (or backlog size) increases to infinity; the latter applies to any reasonable inventory cost structure in which the loss associated with a stockout exceeds the unit's purchase price. Such assumption is to ensure the existence of finite order-up-to level for each level of sales effort. The second part is a technical assumption to ensures the existence of the optimal policy. Actually, linear inventory holding and backlogging cost setting, that is, $H_{t}(I)=h_{t} I^{+}+b_{t} I^{-}\left(h_{t}\right.$ is the linear holding cost and $b_{t}$ is the linear backlogging cost), which is widely adopted in literature, satisfies this assumption. From this assumption, it is a direct result that $L_{t}(y, e)$ is jointly convex in $(y, e)$.

Now, we begin to develop the multi period dynamic programming model for this finite horizon planning problem. Let $\pi_{t}^{*}\left(x_{t}\right)$ denote maximum expected discounted profit when starting period $t$ with state $x$, then, the optimality equation of this finite horizon problem is

$$
\pi_{t}^{*}\left(x_{t}\right)=c_{t} x_{t}+\max _{y_{t} \geq x_{t}, e_{t} \in\left[e_{\min }, e_{\max }\right]} J_{t}^{\prime}\left(y_{t}, e_{t}\right),
$$

where

$$
\begin{aligned}
J_{t}^{\prime}\left(y_{t}, e_{t}\right)= & p_{t} E\left[e_{t} \xi_{t}\right]-c_{t} y_{t}-L_{t}\left(y_{t}, e_{t}\right) \\
& -v_{t}\left(e_{t}\right)+\alpha E\left[\pi_{t+1}^{*}\left(y_{t}-e_{t} \xi_{t}\right)\right]
\end{aligned}
$$

and $\alpha \leq 1$ is the discount factor for future periods. In the above formula, $p_{t} E\left[e_{t} \xi_{t}\right]$ is the revenue collected in this period. Thus, we implicitly assume that we still collect money today for the backlog demand. Without loss of generality, we assume $\pi_{T+1}^{*}\left(x_{T+1}\right)=c_{T+1} x_{T+1}$; that is, all demand at the end of the planning horizon will be satisfied at the unit $\operatorname{cost} c_{T+1}$ and all leftover inventory is salvaged at this unit cost.

To ease analysis, let $\Pi_{t}^{*}\left(x_{t}\right)=\pi_{t}^{*}\left(x_{t}\right)-c_{t} x_{t}$. Then, $\Pi_{T+1}^{*}\left(x_{T+1}\right)=0$, and the original dynamic programming problem can be reformulated as follows:

$$
\Pi_{t}^{*}\left(x_{t}\right)=\max _{y_{t} \geq x_{t}, e_{t} \in\left[e_{\min }, e_{\max }\right]} J_{t}\left(y_{t}, e_{t}\right),
$$

where

$$
\begin{aligned}
J_{t}\left(y_{t}, e_{t}\right)= & p_{t} E\left[e_{t} \xi_{t}\right]-c_{t} y_{t}-L_{t}\left(y_{t}, e_{t}\right)-v_{t}\left(e_{t}\right) \\
& +\alpha c_{t+1} E\left[y_{t}-e_{t} \xi_{t}\right]+\alpha E\left[\Pi_{t+1}^{*}\left(y_{t}-e_{t} \xi_{t}\right)\right] \\
= & \left(p_{t}-\alpha c_{t+1}\right) e_{t} \mu_{t}-\left(c_{t}-\alpha c_{t+1}\right) y_{t}-L_{t}\left(y_{t}, e_{t}\right) \\
& -v_{t}\left(e_{t}\right)+\alpha E\left[\Pi_{t+1}^{*}\left(y_{t}-e_{t} \xi_{t}\right)\right] .
\end{aligned}
$$

Thus, the inner part of the objective function, that is, $J_{t}\left(y_{t}, e_{t}\right)$, is called the profit-to-go function and is independent of the initial inventory level $x_{t}$. The following analysis will be based on the value function $\Pi_{t}^{*}(x)$ instead of $\pi_{t}^{*}(x)$.

Before further analyzing the optimal policy, we first define the so-called $(s, S, e)$ policy as follows.

Definition 2. An $(s, S, e)$ policy is characterized by two stock levels $\left(s_{t}^{*}, S_{t}^{*}\right)$ and a list sales effort $e_{t}^{*}$. If the inventory level is below the stock level $s_{t}^{*}$, it is increased to the stock level $S_{t}^{*}$ and the sales effort $e_{t}^{*}$ is exerted. If the inventory level is above the stock level $s_{t}^{*}$, then, nothing is ordered but only sales effort is exerted. In addition, the sales effort level is nondecreasing in the initial inventory level.

When $s_{t}^{*}=S_{t}^{*}\left(\equiv y_{t}^{*}\right)$, this policy is reduced the so-called base stock list sales effort policy, which is similar to the base stock list price policy proposed by Federgruen and Heching [5]. As we will show in the following analysis, when there is no setup cost for procurement, that is, the model (1), a base stock list sales effort policy is optimal, while when there is a fixed setup cost for each procurement, the $(s, S, e)$ policy is optimal.

2.1. Analysis of the Finite Horizon Model. Before we derive the optimal policy, we first present the following result on the properties of the value function and the profit-to-go function.

Theorem 3. For any $t=1,2, \ldots, T$, the function $J_{t}\left(y_{t}, e_{t}\right)$ is jointly concave in $y_{t}$ and $e_{t}$. Moreover, the function $\Pi_{t}^{*}\left(x_{t}\right)$ is concave and nondecreasing in $x_{t}$.

Proof. The proof will be done by induction. It is easy to verify $J_{T}(\cdot, \cdot)$ is jointly concave. Now, by assuming $J_{t+1}\left(y_{t+1}, e_{t+1}\right)$ is jointly concave in $y_{t+1}$ and $e_{t+1}$, we will show that the property holds even for $J_{t}\left(y_{t}, e_{t}\right)$, which is given by

$$
\begin{aligned}
J_{t}\left(y_{t}, e_{t}\right)= & \left(p_{t}-\alpha c_{t+1}\right) e_{t} \mu_{t}-\left(c_{t}-\alpha c_{t+1}\right) y_{t}-L_{t}\left(y_{t}, e_{t}\right) \\
& -v_{t}\left(e_{t}\right)+\alpha E\left[\Pi_{t+1}^{*}\left(y_{t}-e_{t} \xi_{t}\right)\right] .
\end{aligned}
$$

It is straightforward to verify that $\left(p_{t}-\alpha c_{t+1}\right) e_{t} \mu_{t}$ is jointly concave in $p_{t}$ and $e_{t}$. For $E\left[\Pi_{t+1}^{*}\left(y_{t}-e_{t} \xi_{t}\right)\right]$, let $\lambda \in[0,1]$ and consider two pairs $\left(y_{t}^{(1)}, e_{t}^{(1)}\right)$ and $\left(y_{t}^{(2)}, e_{t}^{(2)}\right)$, then, we get

$$
\begin{aligned}
\Pi_{t+1}^{*} & {\left[\lambda y_{t}^{(1)}+(1-\lambda) y^{(2)}-\left(\lambda e_{t}^{(1)}+(1-\lambda) e_{t}^{(2)}\right) \xi_{t}\right] } \\
& =\Pi_{t+1}^{*}\left[\lambda\left(y^{(1)}-e_{t}^{(1)} \xi_{t}\right)+(1-\lambda)\left(y_{t}^{(2)}-e_{t}^{(2)} \xi_{t}\right)\right] \\
& \geq \lambda \Pi_{t+1}^{*}\left[y^{(1)}-e_{t}^{(1)} \xi_{t}\right]+(1-\lambda) \Pi_{t+1}^{*}\left(y_{t}^{(2)}-e_{t}^{(2)} \xi_{t}\right) .
\end{aligned}
$$

The above inequality holds since $\Pi_{t+1}^{*}(\cdot)$ is concave. This implies that $E\left[\Pi_{t+1}^{*}\left(y_{t}-e_{t} \xi_{t}\right)\right]$ is jointly concave in $y_{t}$ and $e_{t}$. As the other terms of $j_{t}\left(y_{t}, e_{t}\right)$ are jointly concave in $y_{t}$ and $e_{t}$, then $J_{t}\left(y_{t}, e_{t}\right)$ is jointly concave in $y_{t}$ and $e_{t}$. The concavity and monotonicity of $\Pi_{t}^{*}(\cdot)$ is immediate.

This theorem indicates the concavity of the value function and the existence of the optimal solution to the maximization problem. Thus, we can define the values $\left(y_{t}^{*}, e_{t}^{*}\right)$ and $e^{*}(x)$ as follows:

$$
\begin{aligned}
\left(y_{t}^{*}, e_{t}^{*}\right) & =\arg \max _{\left(y_{t}, e_{t}\right)}\left\{J_{t}\left(y_{t}, e_{t}\right)\right\}, \\
e^{*}(x) & =\arg \max _{e_{t}}\left\{J_{t}\left(x, e_{t}\right)\right\} .
\end{aligned}
$$


Then, the above theorem shows that whenever the inventory level is less than $y_{t}^{*}$, the retailer should order to bring the inventory up to $y_{t}^{*}$ and exert sales effort $e_{t}^{*}$. However, to show that the optimal policy is a base stock list sales effort policy, we still need to show that the optimal sales effort is increasing in the initial inventory level. This will be stated by the following theorem.

Theorem 4. For anyt $=1,2, \ldots, T$,

(1) the optimal sales effort $e_{t}^{*}(x)$ is increasing in $x$ with $e_{t}^{*}(x) \geq e_{t}^{*}$

(2) a base stock list sales effort with base stock $y_{t}^{*}$ and $e_{t}^{*}$ is optimal.

Proof. (1) We first show that $J_{t}\left(y_{t}, e_{t}\right)$ is supermodular. Since the sum of supermodular function is supermodular, it suffices to establish supermodularity for each term of $J_{t}\left(y_{t}, e_{t}\right)$.

Because $\partial^{2}\left(p_{t}-\alpha c_{t+1}\right) e_{t} \mu_{t} / \partial y_{t} \partial e_{t} \geq 0$, then, $p_{t} E\left[\min \left(y_{t}, e_{t} \xi_{t}\right)\right]$ is supermodular. Consider $-L_{t}\left(y_{t}, e_{t}\right)=$ $-E\left[H_{t}\left(y_{t}-e_{t} \xi_{t}\right)\right]$,

$$
-\frac{\partial^{2} H_{t}\left(y_{t}-e_{t} \xi_{t}\right)}{\partial y_{t} \partial e_{t}}=H^{\prime \prime} \xi_{t} \geq 0 .
$$

So, $\left(\partial^{2}-L_{t}\left(y_{t}-e_{t} \xi_{t}\right)\right) / \partial y_{t} \partial e_{t} \geq 0$; thus, $L_{t}(\cdot, \cdot)$ is supermodular.

To show $E\left[\Pi_{t+1}^{*}\left(y_{t}-e_{t} \xi_{t}\right)\right]$ is supermodular, fix $\xi_{t}$ and consider an arbitrary pair of inventory level $\left(y_{t}^{(1)}, y_{t}^{(2)}\right)$ and any pair of sales effort levels $\left(e_{t}^{(1)}, e_{t}^{(2)}\right)$ with $y_{t}^{(1)} \geq y_{t}^{(2)}$ and $e_{t}^{(1)} \geq e_{t}^{(2)}$. By the concavity and monotonicity of $\Pi_{t}^{*}(\cdot)$,

$$
\begin{aligned}
& \Pi_{t}^{*}\left(y^{(1)}-e^{(1)} \xi_{t}\right)-\Pi_{t}^{*}\left(y^{(2)}-e^{(1)} \xi_{t}\right) \\
& \quad \geq \Pi_{t}^{*}\left(y^{(1)}-e^{(2)} \xi_{t}\right)-\Pi_{t}^{*}\left(y^{(2)}-e^{(2)} \xi_{t}\right),
\end{aligned}
$$

that is, $\Pi_{t}^{*}\left(y^{(1)}-e^{(1)} \xi_{t}\right)+\Pi_{t}^{*}\left(y^{(2)}-e^{(2)} \xi_{t}\right) \geq \Pi_{t}^{*}\left(y^{(2)}-e^{(1)} \xi_{t}\right)+$ $\Pi_{t}^{*}\left(y^{(1)}-e^{(2)} \xi_{t}\right)$, because concave function has decreasing difference. Then, $E\left[\Pi_{t+1}^{*}\left(y_{t}-e_{t} \xi_{t}\right)\right]$ is supermodular in $y_{t}$ and $e_{t}$.

The other terms of $J_{t}\left(y_{t}, e_{t}\right)$ are supermodular obviously. Thus, we obtain that $J_{t}\left(y_{t}, e_{t}\right)$ is supermodular. Then, by Theorem 2.3 in Vives [17], $e_{t}^{*}(x)$ is increasing in $x$.

(2) Immediate from part (1) and Theorem 3.

Federgruen and Heching [5] show that the optimal selling price $p^{*}(x)$ is nonincreasing in $x$; that is, when the beginning inventory is too high, the retailer will choose a lower price to achieve a higher demand. Our result has the similar principle: when the beginning inventory is too high, the retailer will exert more sales effort in order for high demand.

Remark 5. We can see from the proofs that the structure of the optimal policy does not depend on the terminal value $\pi_{T+1}^{*}(x)=c_{T+1} x$. It only requires that $\Pi_{T+1}^{*}(x)=$ $\pi_{T+1}^{*}(x)-c_{T+1} x$ is concave and decreasing in $x$. For example, when all unsatisfied demand is lost and all leftover inventory is salvaged at zero, that is, the terminal value $\pi_{T+1}^{*}(x)=$ $\gamma \min \{x, 0\}$ in which $\gamma>\max _{t} p_{t}$ is the shortage cost, we have $c_{T+1}=\gamma$ and $\Pi_{T+1}^{*}(x)=\gamma \min \{-x, 0\}$, which is also concave and nonincreasing in $x$. Thus, the results still hold. Moreover, in the case of $\pi_{T+1}^{*}(x)=c_{T+1} x$, we can prove that the myopic policy is optimal when the system parameters are stationary.

\section{Extension}

So far, we have established analytical results associated with the sales efforts demand, in which the ordering cost is proportional to the order quantity and the order size is continuous in the sense that the retailer can order any quantity he/she wishes. However, in practice, the placement of an order always incurs additional transaction cost other than the cost of the product, for example, the cost of paper work, the cost of transhipment, and so forth. These transaction costs, or setup cost, are not neglectable in most times. Moreover, in some cases, there may be some constraints on the order amount the retailer should order for each transaction; for example, each order should be multiple of certain fixed amount. In this section, we extend our analysis to the combination of sales effort and inventory management with fixed order cost and batch orders.

3.1. Combining Sales Effort and Inventory Control with Setup Cost. Now, we first extend the analysis in the last section to the case where there is a setup cost for each order; that is, when we replenish the inventory, we need to pay the fixed order cost besides variable order cost. Keeping other notations unchanged, let $k_{t}$ be fixed order cost at the beginning of period $t$. Then, when the order-up-to level $y_{t}>$ $x_{t}$, a cost $k_{t}$ occurs in addition to the variable cost, and when $y_{t} \leq x_{t}$, no procurement cost occurs. To make the problem tractable, we need the following assumption on the relationship between $k_{t}, t=1,2, \ldots, T$.

Assumption 6. For each period $0 \leq t \leq T, k_{t} \geq \alpha k_{t+1}$.

This assumption means that the discounted setup cost in future periods is less than the current setup cost. The case when the setup costs are constant among periods satisfies this assumption.

Then, we can formulate this problem as follows:

$$
\pi_{t}^{*}\left(x_{t}\right)=c_{t} x_{t}+\max _{y_{t} \geq x_{t}, e_{t} \in\left[e_{\min }, e_{\max }\right]}-k_{t} \delta\left(y_{t}-x_{t}\right)+J_{t}\left(y_{t}, e_{t}\right),
$$

where

$$
\begin{aligned}
J_{t}\left(y_{t}, e_{t}\right)= & p_{t} E\left[e_{t} \xi_{t}\right]-c_{t} y_{t}-L_{t}\left(y_{t}, e_{t}\right) \\
& -v_{t}\left(e_{t}\right)+\alpha E\left[\pi_{t+1}^{*}\left(y_{t}-e_{t} \xi_{t}\right)\right]
\end{aligned}
$$

With the definition of $K$-convex widely studied in traditional inventory literature (see, e.g., Scarf [18] and Veinott [19]), we can get the following results associating with the properties of the value function and the resulting optimal policy. 
Theorem 7. (a) For $t=1,2, \ldots, T, J_{t}\left(y_{t}, e_{t}\right)$ is continuous in $\left(y_{t}, e_{t}\right)$ and $\lim _{|y| \rightarrow \infty} J_{t}\left(y_{t}, e_{t}\right)=-\infty$ for any $e_{t} \in\left[e_{\min }, e_{\max }\right]$. Hence, for any fixed $y_{t}$, there is a finite best sales effort $e_{t}^{*}\left(y_{t}\right)$.

(b) For $t=1,2, \ldots, T$, both $J_{t}\left(y_{t}, e_{t}^{*}\left(y_{t}\right)\right)$ and $\pi_{t}^{*}\left(x_{t}\right)$ are $k_{t}$-concave.

(c) For $t=1,2, \ldots, T$, there exist $s_{t}$ and $S_{t}$ with $s_{t} \leq S_{t}$ such that it is optimal to order $S_{t}-x_{t}$ and exert sales effort $e_{t}^{*}\left(S_{t}\right)$ when $x_{t}<s_{t}$; otherwise, do not order and exert sales effort $e_{t}^{*}\left(x_{t}\right)$.

Proof. We will prove this theorem by induction. Assume parts (a), (b), and (c) hold for $t+1$. Then, as it is easy to check that $J_{t}\left(y_{t}, e_{t}\right)$ is continuous, there is an optimal sales effort $e_{t}^{*}\left(y_{t}\right)$ for any fixed $y_{t}$.

We now focus on part (b). We will show that both $J_{t}\left(y_{t} \cdot e^{*}\left(y_{t}\right)\right)$ and $\pi^{*}\left(x_{t}\right)$ are $k_{t}$-concave.

For any $y \leq y^{\prime}$ and $\lambda \in[0,1]$, we have $\pi_{t+1}^{*}$ is $k_{t+1}{ }^{-}$ concave. Then,

$$
\begin{aligned}
\pi_{t+1}^{*}( & \left.\lambda\left(y-e_{t}^{*}(y) \xi_{t}\right)+(1-\lambda)\left(y^{\prime}-e_{t}^{*}\left(y^{\prime}\right) \xi_{t}\right)\right) \\
\geq & \lambda \pi_{t+1}^{*}\left(y-e_{t}^{*}(y) \xi_{t}\right) \\
& +(1-\lambda) \pi_{t+1}^{*}\left(y^{\prime}-e_{t}^{*}\left(y^{\prime}\right) \xi_{t}\right)-(1-\lambda) k_{t+1} .
\end{aligned}
$$

Because $H_{t}$ is convex, and $p_{t} E\left[e_{t} \xi_{t}\right]-L_{t}\left(y_{t}, e_{t}\right)=p_{t} e_{t} \mu_{t}-$ $E H\left(y_{t}-e_{t} \xi_{t}\right):=L^{+}\left(y_{t}, e_{t}\right)$, it is easy to verify that $L^{+}(\cdot, \cdot)$ is jointly concave. Then,

$$
\begin{aligned}
& L^{+}\left(\lambda y+(1-\lambda) y^{\prime}, \lambda e^{*}(y)+(1-\lambda) e^{*}\left(y^{\prime}\right)\right) \\
& \quad \geq \lambda L^{+}\left(y, e^{*}(y)\right)+(1-\lambda) L^{+}\left(y^{\prime}, e^{*}\left(y^{\prime}\right)\right) .
\end{aligned}
$$

Because $v(\cdot)$ is convex, then

$$
\begin{aligned}
& v\left(\lambda e_{t}^{*}(y)+(1-\lambda) e^{*}\left(y^{\prime}\right)\right) \\
& \quad \geq \lambda v\left(e^{*}(y)\right)+(1-\lambda) v\left(e^{*}\left(y^{\prime}\right)\right) .
\end{aligned}
$$

Thus, we have proved that

$$
\begin{aligned}
J_{t}(\lambda y & \left.+(1-\lambda) y^{\prime}, \lambda e_{t}^{*}(y)+(1-\lambda) e_{t}^{*}\left(y^{\prime}\right)\right) \\
& \geq \lambda J_{t}\left(y, e_{t}^{*}(y)\right)+(1-\lambda) J_{t}\left(y^{\prime}, e_{t}^{*}\left(y^{\prime}\right)\right)-(1-\lambda) \alpha k_{t+1} .
\end{aligned}
$$

Because $e^{*}\left(\lambda y+(1-\lambda) y^{\prime}\right)$ is the best sales effort for inventory level $\lambda y+(1-\lambda) y^{\prime}$, then

$$
\begin{aligned}
J_{t}(\lambda y & \left.+(1-\lambda) y^{\prime}, e_{t}^{*}\left(\lambda y+(1-\lambda)\left(y^{\prime}\right)\right)\right) \\
& \geq J_{t}\left(\lambda y+(1-\lambda) y^{\prime}, \lambda e_{t}^{*}(y)+(1-\lambda) e_{t}^{*}\left(y^{\prime}\right)\right) .
\end{aligned}
$$

Therefore, $J_{t}\left(y_{t}, e_{t}^{*}\left(y_{t}\right)\right)$ is $\alpha k_{t+1}$-concave, so it is also $k_{t}{ }^{-}$ concave, because of Assumption 6.

Thus, there exist $s_{t}<S_{t}$, such that $S_{t}$ maximizes $J_{t}\left(y_{t}\right.$, $\left.e^{*}\left(y_{t}\right)\right)$ and $s_{t}$ is the smallest value of $y$, such that

$$
\begin{aligned}
J_{t}\left(S_{t}, e_{t}^{*}\left(S_{t}\right)\right) & =J_{t}\left(y, e_{t}^{*}(y)\right)-k_{t}, \text { and } \\
\pi_{t}^{*}\left(x_{t}\right) & = \begin{cases}-k+J_{t}\left(S_{t}, e_{t}^{*}\left(S_{t}\right)\right)+c_{t} x & \text { if } x \leq s_{t}, \\
J_{t}\left(x_{t}, e_{t}^{*}\left(x_{t}\right)\right)+c_{t} x & \text { if } x \geq s_{t} .\end{cases}
\end{aligned}
$$

The $k_{t}$-concavity of $\pi_{t}^{*}\left(x_{t}\right)$ can be checked directly from $k_{t}$-concavity of $J_{t}\left(y_{t}, e_{t}^{*}\left(y_{t}\right)\right)$; see Zipkin [20] for a proof.

3.2. Combining Sales Effort and Batch Ordering. In this section, we will extend the analysis of the last section to the case where each order is in batch size. That is, orders must be placed in multiples of some standard batch size $q$, for example, a case, a barrel, or a truck load. To make the problem tractable, we assume the cost parameters are stationary, because even for a general inventory problem, a $(r, N q)$ policy is not optimal when the parameters are nonstationary. Let $c_{1}=c_{2}=\cdots=c_{T+1}=c, p_{1}=p_{2}=\cdots=$ $p_{T+1}=p$, and $L_{1}(\cdot, \cdot)=L_{2}(\cdot, \cdot)=\cdots=L_{T+1}(\cdot, \cdot)=L(\cdot, \cdot)$. Therefore, for any policy $\Theta$, the total discounted expected profit for this finite horizon dynamic programming problem is

$$
\begin{aligned}
\pi^{(T)}\left(x_{0} \mid \Theta\right)=\sum_{t=0}^{t=T} \alpha^{t}\left[-c\left(y_{t}-x_{t}\right)+p E\left[e_{t} \xi_{t}\right]\right. \\
\left.\quad-L\left(y_{t}, e_{t}\right)-v\left(e_{t}\right)\right]+\alpha^{T+1} c x_{T+1} \\
=c x_{0}+\sum_{t=1}^{t=T} \alpha^{t}\left[-(1-\alpha) c y_{t}+(p-\alpha c) e_{t} \mu_{t}\right. \\
=c x_{0}+\sum_{t=1}^{t=T} C^{+}\left(y_{t}, e_{t}\right),
\end{aligned}
$$

where $x_{0}$ is initial inventory and

$$
C^{+}(y, e)=-(1-\alpha) c y+(p-\alpha c) e \mu-L(y, e)-v(e) .
$$

Then, we can get the following result for the joint optimal inventory and sales effort policy when each order is in batch size.

Theorem 8. (1) $C^{+}(y, e)$ is jointly concave in $y$ and $e$.

(2) $C^{+}(y, e)$ is continuous in $y$ and $e$ and $\lim _{|y| \rightarrow \infty}\left(C^{+}(y, e)\right)=-\infty$ for any fixed $e \in\left[e_{\min }, e_{\max }\right]$. Hence, for any fixed $y, C^{+}(y, e)$ has a finite maximizer, denoted by $e^{*}(y)$, which is increasing in $y$, and $C^{+}\left(y, e^{*}(y)\right)$ is concave in $y$.

(3) The optimal policy is in a $(r, N q, e)$ type; that is, if the inventory at the beginning of each period is lower than $r$, then order minimum multiple of $q$, such that the inventory level $y$ after ordering is greater than $r$, and exert sales effort $e^{*}(y)$; otherwise, do not order and exert sales effort $e^{*}(x)$.

Proof. (1) Recalling Theorem $3, C^{+}(y, e)$ is jointly concave in $y$ and $e$. 
TABLE 1: Basic parameter settings.

\begin{tabular}{lccccccc}
\hline Demand Dist. & $c$ & $b$ & $h$ & $a_{1}$ & $a_{2}$ & $p$ & $\alpha$ \\
\hline$\mu=100, \sigma=20$ & 10 & 10 & 5 & 400 & 0 & 30 \\
\hline
\end{tabular}

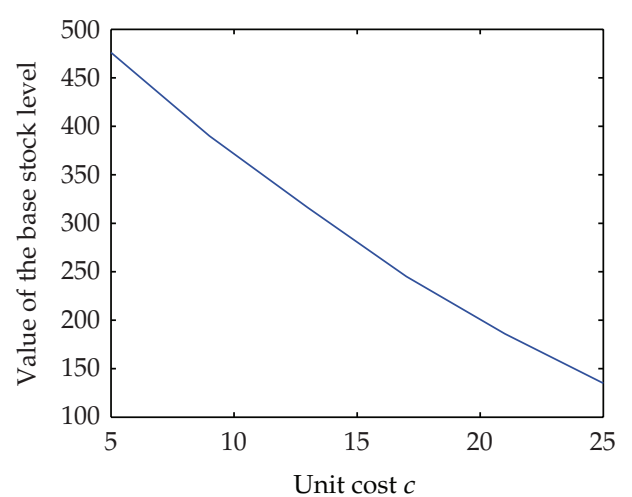

(a) Base stock level

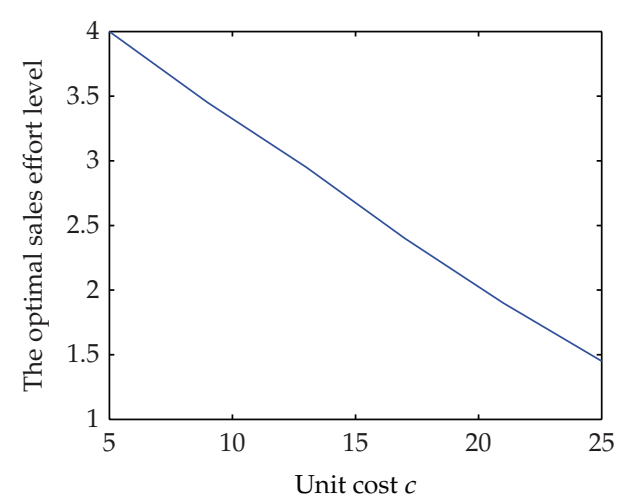

(b) Optimal sales effort level

Figure 1: An illustration of the optimal policies with respect to unit cost.

(2) From part (a), for any fixed $y$, there is an optimal sales effort $e^{*}(y)$. For the monotony of $e^{*}(y)$, consider the crossderivative of $C^{+}(y, e)$,

$$
\begin{aligned}
\frac{\partial^{2} C^{+}(y, e)}{\partial y \partial e} & =\frac{\partial^{2}(p-\alpha c) e \mu}{\partial y \partial e}-\frac{\partial^{2} L(y, e)}{\partial y \partial e} \\
& =\mu+E\left[H^{\prime \prime}(y-e \xi) \xi\right] \geq 0 .
\end{aligned}
$$

Thus, $C^{+}(y, e)$ is supermodular in $y$ and $e$. So, $e^{*}(y)$ is increasing in $y$.

For the concavity of $C^{+}\left(y, e^{*}(y)\right)$, we consider

$$
\begin{aligned}
C^{+}\left(\alpha y_{1}+(1-\alpha) y_{2}, e^{*}\left(\alpha y_{1}+(1-\alpha) y_{2}\right)\right) \\
\quad \geq C^{+}\left(\alpha y_{1}+(1-\alpha) y_{2}, \alpha e^{*}\left(y_{1}\right)+(1-\alpha) e^{*}\left(y_{2}\right)\right) \\
\geq \alpha C^{+}\left(y_{1}, e^{*}\left(y_{1}\right)\right)+(1-\alpha) C^{+}\left(y_{2}, e^{*}\left(y_{2}\right)\right) .
\end{aligned}
$$

The first inequality holds, because $e^{*}\left(\alpha y_{1}+(1-\alpha) y_{2}\right)$ is the best sales effort for inventory level $\alpha y_{1}+(1-\alpha) y_{2}$; the second inequality holds, because of $C^{+}(y, e)$ 's joint concavity.

(3) Let $y^{*}=\arg \max C^{+}\left(y, e^{*}(y)\right)$, and choose $r$, such that $C^{+}\left(r, e^{*}(r)\right)=C^{+}\left(r+q, e^{*}(r+q)\right)$ and $r \leq y^{*} \leq r+q$. Denote the $(r, N q, e)$ policy by $\Theta^{*}$. For any other policy $\Theta$, under the same demand sample path, we have $y_{t}^{*}=y_{t}$ or $\left|y_{t}^{*}-y_{t}\right| \geq q$ for any $1 \leq t \leq T$. Then, $C^{+}\left(y_{t}^{*}, p^{*}\left(y_{t}^{*}\right)\right) \geq$ $C^{+}\left(y_{t}, p^{*}\left(y_{t}\right)\right)$ for any $1 \leq t \leq T$. Thus, $\pi^{(T)}\left(x_{0} \mid \Theta^{*}\right) \geq$ $\pi^{(T)}\left(x_{0} \mid \Theta\right)$. Finally, $\Theta^{*}$ is an optimal policy.

\section{Numerical Study}

In this section, basing on model (1), we report a numerical study conducted to attain qualitative insights into the structure of optimal policies and their sensitivity with respect to several parameters. Among the major questions investigated, we focus in particular on how the optimal decisions vary against system parameters, that is, the demand uncertainty, the unit cost, and the sales effort cost.

Before conducting the numerical studies, we first setup the configuration of the basic model. The single period inventory holding and backlogging cost setting is $H_{t}(I)=$ $h_{t} I^{+}+b_{t} I^{-}$. The system is assumed to run in a stationary setting under a planning horizon with 5 periods, that is, $T=$ $5, h_{1}=\cdots h_{T}=h, p_{1}=\cdots p_{T}=p, c_{1}=\cdots c_{T}=c$, and $b_{1}=\cdots b_{T}=b$. The sales effort cost function $v(e)=a_{1}(e-1)^{2}+$ $a_{2}(e-1)$ for $6 \geq e \geq 1$, in which $a_{1}$ measures the diseconomy of scale of the sales effort level. This sales effort function is sufficient for us to study the impact of cost parameters in our problem as we can approximate other forms of functions by a quadratic function as a direct result of Taylor expansion. This setting appears in several literature references, for example, Taylor [21]. The random part of the demand, $\xi$, is assumed to follow a normal distribution, which is a typical setting both in practice and academy. The values of the system parameters are provided in Table 1.

Figures 1(a) and 1(b) illustrate that both the optimal base stock level and the sales effort level decrease as the unit cost increases. This is rather intuitive as the high unit cost reduces the retailer's motivation to order more, which results in less sales effort exerted.

Figures 2(a) and 2(b) illustrate a similar pattern for the sales effort cost parameters $a_{1}$. However, the undermining reason is different. In this case, the high sales effort cost reduces the incentives to stimulate demand, which results in the low base stock level.

Figures 3(a) and 3(b) illustrate the effect of demand uncertainty on the optimal policies. Interestingly, it shows that when the demand uncertainty becomes large, the optimal order-up-to level is large too. The reason is that when the shortage penalty is high, given the inventory level, large 


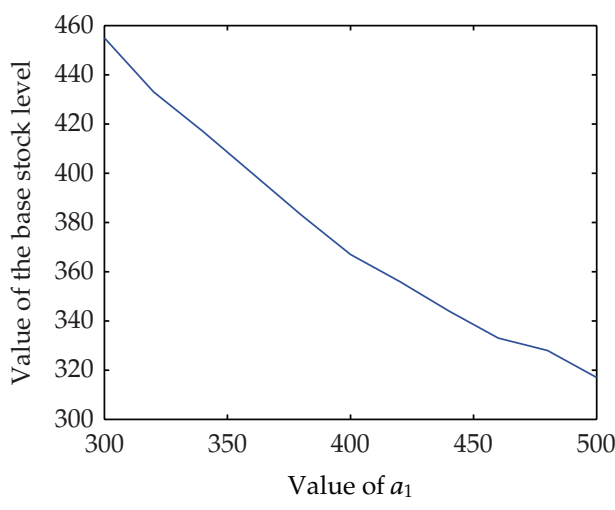

(a) Base stock level

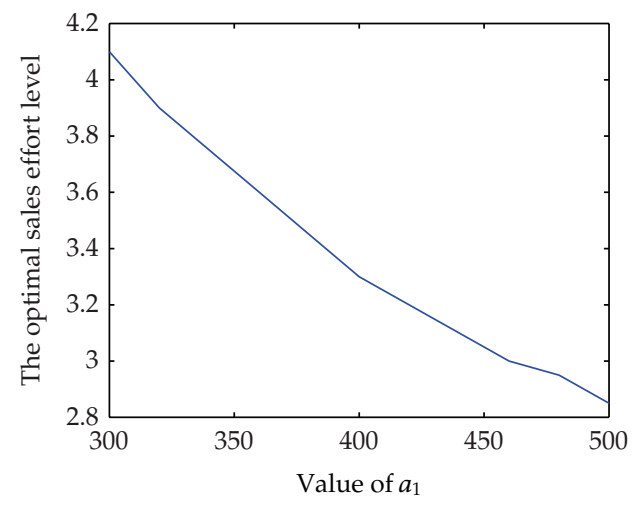

(b) Optimal sales effort leve

FIGURE 2: An illustration of the optimal policies with respect to sales effort cost.

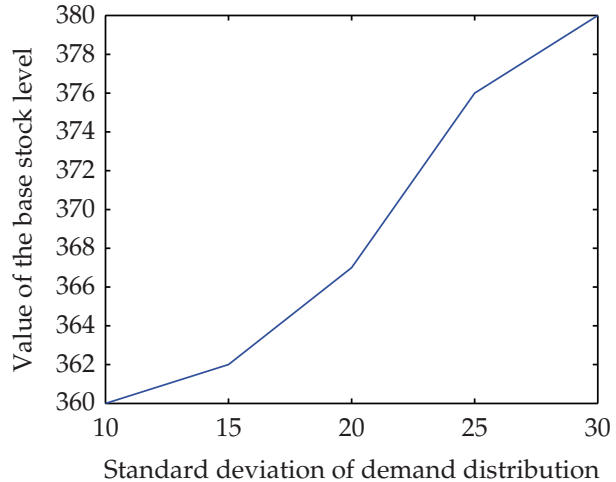

(a) Base stock level

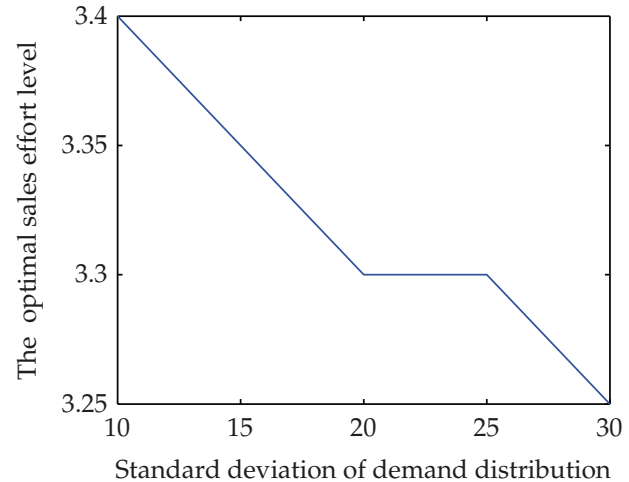

(b) Optimal sales effort level

FIGURE 3: An illustration of the optimal policies with respect to demand uncertainty.

demand uncertainty will result in high penalty cost, which induce the retailer to order more to avoid such shortage. However, the numerical results show that the optimal sales effort level is decreasing in the demand uncertainty. This is because, when the demand has a multiplicative form with the sales effort level, high sales effort level also induces high variance of the demand distribution, although it increases the expected demand; that is, the cost caused by additional demand variance is larger than the profits made by additional expected demand.

\section{Conclusion}

In this paper, we study joint inventory and sales effort management of a retailer in a broad context and investigate optimal policies for a single item, periodic-review system. The retailer decides on the order replenishment and sales effort level jointly in each period, in which the demand has a multiplicative form with the sales effort level. His objective is to find a joint optimal inventory replenishment and sales effort policy to maximize the discounted profit over a finite horizon. We consider three cases depending on the cost structure and the constrains on order quantity: linear ordering cost without setup cost, linear ordering cost with setup cost, and batch size for each ordering. Through our analysis, we find that a base stock list sales effort policy, an $(s, S, e)$ policy, and a $(r, N q, e)$ policy are optimal for these three cases, respectively. Numerical studies show that the base stock level and the optimal sales effort level are decreasing with the unit cost and the diseconomy of scale for the sales effort. However, although the base stock level is increasing in the demand uncertainty, the corresponding sales effort level is decreasing, which implies that the cost caused by additional demand variance is larger than the profits made by additional expected demand.

Several extensions based on our model can be analyzed in future research. First, models should be developed to address the joint consideration on the price, sales effort, and inventory decisions. In our paper, we assume that the selling price is exogenously determined and provide some reasonable arguments. However, in practices, both the price change and the sales effort are exerted, especially in the $\mathrm{B} 2 \mathrm{C} \mathrm{e-commence}$ industry. In this case, why does the retailer take both actions? what is the joint effect of pricing and sales effort decisions on the optimal inventory decisions? Second, the financial state of the retailer and its effect on the optimal decisions should be considered. In our analysis, we implicitly assume the retailer has enough cash on hand to make procurement and sales 
effort decisions. However, in practice, the working capital is always limited. In this case, how should the retailer allocate these limited resources between marketing and inventory? The effect of financial states on the optimal policy is worth investigation.

\section{Acknowledgments}

The authors acknowledge the support of National Natural Science Foundation of China with Research Fund no. 71171105 and no. 70932003, and the China Postdoctoral Science Foundation no. 2012M521054.

\section{References}

[1] C. A. Yano and S. M. Gilbert, "Coordinated pricing and production/procurement decisions: a review," in Managing Business Interfaces: Marketing, Engineering and Manufacturing Perspectives, A. Chakravarty and J. Eliashberg, Eds., Kluwer Academic Publishers, Boston, Mass, USA, 2004.

[2] M. Karakul, "Joint pricing and procurement of fashion products in the existence of clearance markets," International Journal of Production Economics, vol. 114, no. 2, pp. 487-506, 2008.

[3] D. A. Serel, "Inventory and pricing decisions in a singleperiod problem involving risky supply," International Journal of Production Economics, vol. 116, no. 1, pp. 115-128, 2008.

[4] S. Webster and Z. K. Weng, "Ordering and pricing policies in a manufacturing and distribution supply chain for fashion products," International Journal of Production Economics, vol. 114, no. 2, pp. 476-486, 2008.

[5] A. Federgruen and A. Heching, "Combined pricing and inventory control under uncertainty," Operations Research, vol. 47, no. 3, pp. 454-475, 1999.

[6] X. Chen and D. Simchi-Levi, "Coordinating inventory control and pricing strategies with random demand and fixed ordering cost: the finite horizon case," Operations Research, vol. 52, no. 6, pp. 887-896, 2004.

[7] X. Chen and D. Simchi-Levi, "Coordinating inventory control and pricing strategies with random demand and fixed ordering cost: the infinite horizon case," Mathematics of Operations Research, vol. 29, no. 3, pp. 698-723, 2004.

[8] X. Chen, X. Zhou, and Y. Chen, "Integration of inventory and pricing decisions with costly price adjustments," Operations Research, vol. 59, pp. 1144-1158, 2011.

[9] Y. Balcer, "Partially controlled demand and inventory control: an additive model," Navel Research Logistics, vol. 27, no. 2, pp. 273-288, 1980.

[10] F. Cheng and S. P. Sethi, "Periodic review inventory model with demand influenced by promotion decisions," Management Science, vol. 45, no. 11, pp. 1510-1523, 1999.

[11] E. L. Porteus and S. Whang, "On manufacturing/marketing incentives," Management Science, vol. 37, no. 9, pp. 1166-1181, 1991.

[12] F. Chen, "Sales-force incentives and inventory management," Manufacturing and Service Operations Management, vol. 2, no. 2, pp. 186-202, 2000.

[13] R. Ernst and P. Kouvelis, "Effects of selling packaged goods on inventory decisions," Management Science, vol. 45, no. 8, pp. 1142-1155, 1999.
[14] F. Y. Chen, Y. Feng, and J. Ou, "Management of inventory replenishment and available offerings for goods sold with optional value-added packages," IIE Transactions, vol. 37, no. 5, pp. 397-406, 2005.

[15] J. L. Zhang, J. Chen, and C. Y. Lee, "Joint optimization on pricing, promotion and inventory control with stochastic demand," International Journal of Production Economics, vol. 116, no. 2, pp. 190-198, 2008.

[16] Y. Wei and Y. Chen, "Joint determination of inventory replenishment and sales-effort with uncertain market responses," International Journal of Productions Economics, vol. 134, pp. 368-374, 2011.

[17] X. Vives, Oligopoly Pricing: Old Ideas and New Tools, MIT Press, Cambridge, Mass, USA, 1999.

[18] H. Scarf, "The optimality of $(s, S)$ polices in the dynamic inventory problem," in Mathematical Methods in Social Science, K. Arrow, S. Karlin, and P. Suppes, Eds., Chapter 13, Stanford University Press, 1960.

[19] J. Veinott, "On the opimality of $(s, S)$ inventory policies: new conditions and a new proof," SIAM Journal on Applied Mathematics, vol. 14, no. 5, pp. 1067-1083, 1966.

[20] P. Zipkin, Foundations of Inventory Management, Irwin/ McGraw-Hill Press, 2000.

[21] T. A. Taylor, "Supply chain coordination under channel rebates with sales effort effects," Management Science, vol. 48, no. 8, pp. 992-1007, 2002. 


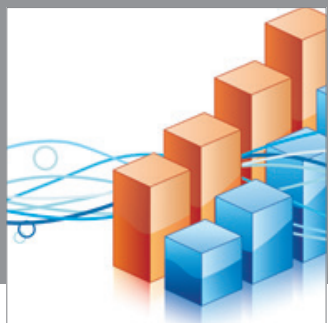

Advances in

Operations Research

mansans

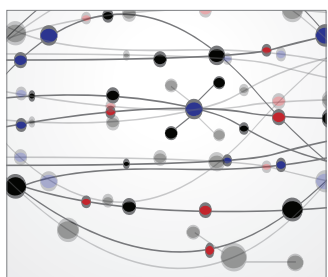

The Scientific World Journal
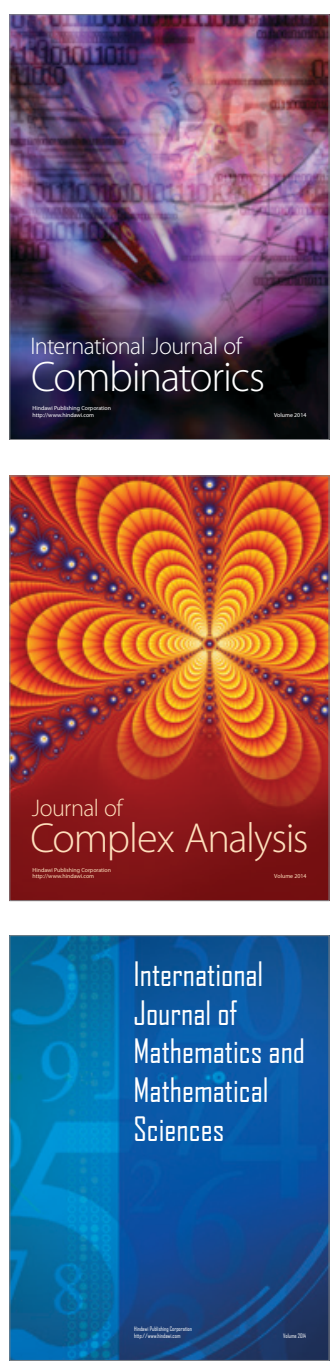
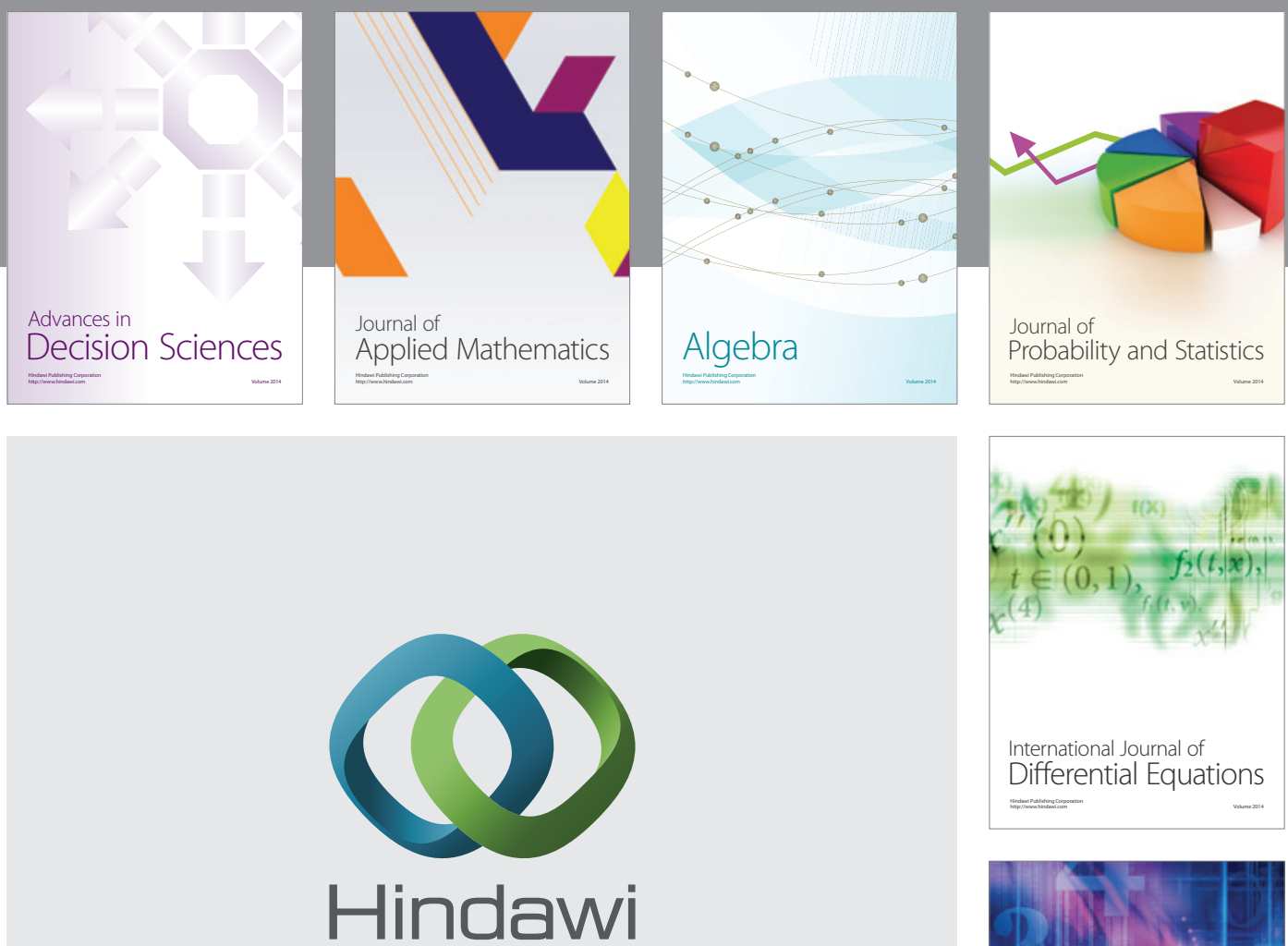

Submit your manuscripts at http://www.hindawi.com
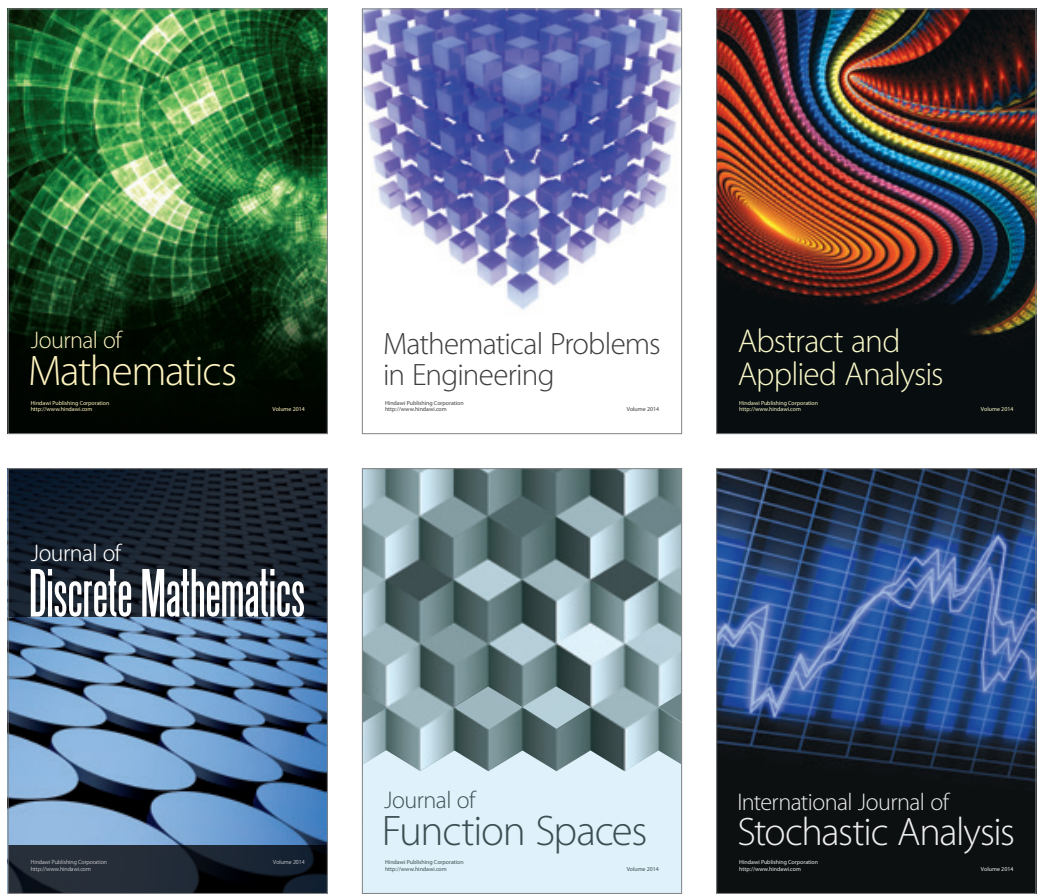

Journal of

Function Spaces

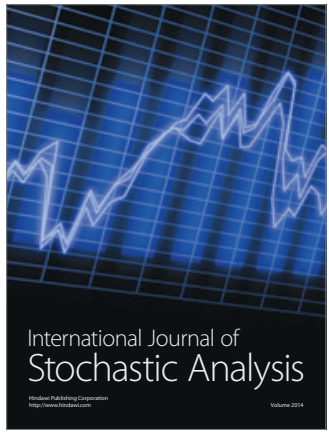

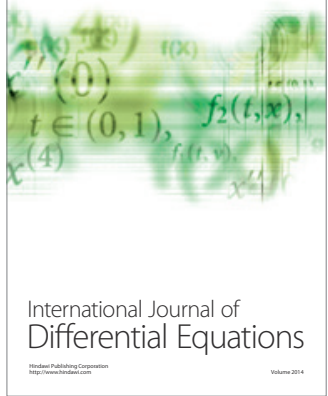
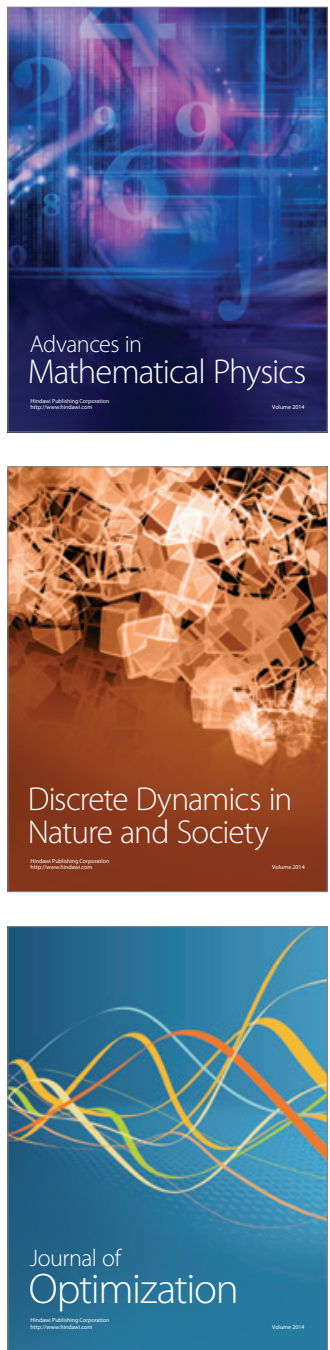